\title{
Estudio de cobertura en anillo para redes de tasa alta
}

\author{
Ashley Meléndez Cano ${ }^{1}$, Sergio Alberto Juárez Cazares ${ }^{1}$, Edgar Allende Chavez ${ }^{2}$, \\ Amit Kumar ${ }^{1}$, José Cruz Núñez Pérez ${ }^{1}$, Andrés Calvillo Téllez ${ }^{1}$ \\ ${ }^{1}$ Instituto Politécnico Nacional, CITEDI, Baja California, \\ México \\ ${ }^{2}$ Instituto Tecnológico de Tijuana, Baja California, \\ México \\ nunez@citedi.mx, calvillo@citedi.mx, edgar.allende@tectijuana.edu.mx
}

\begin{abstract}
Resumen. Se presenta el estudio de cobertura de redes de tasa alta donde lo irregular de la topografía del terreno no permite la viabilidad de conexión por fibra óptica y las condiciones climáticas agreden la calidad de radioenlaces de tasa alta. Además, se establecieron 7 radioenlaces maestro-esclavo en arquitectura de anillo en la región de Tijuana, Baja California para la zona este de la ciudad, estimando mediante simulación de radioenlaces por medio del software sugerido airFiberHD24 con frecuencia de operación en banda libre a 24GHz. Se realizó el cálculo de las pérdidas de propagación en el espacio libre, se obtiene la orientación de las antenas, considerando altura de la antena sobre el nivel del mar, margen de desvanecimiento, y el cálculo de su zona de Fresnel, con lo que se garantiza que la señal se encuentre en el rango debido. Finalmente, se propuso el tipo de antena en base a las especificaciones del fabricante.
\end{abstract}

Palabras clave: cobertura, conectividad inalámbrica, redes de tasa alta.

\section{Study of Ring Coverage for High Rate Networks}

\begin{abstract}
The study of coverage of high-rate networks is presented where irregular terrain topography does not allow the viability of a fiber optic connection and climatic conditions vanished the quality of high-rate radio links. In addition, seven master-slave radio links were established in a ring topology at Tijuana city, Baja California for the eastern zone of the city, we estimated the simulation of radio links using the suggested software airFiberHD24 with the frequency of operation in a free band at $24 \mathrm{GHz}$. We obtained the estimation of the points of view of the elevation of the antennas, the height over the sea level, the fading margin and the calculation of its Fresnel zone, thereby ensuring that the signal is in the proper range. Finally, the type of antenna was proposed based on the manufacturer specifications.
\end{abstract}

Keywords: coverage, high rate networks, wireless connectivity. 


\section{Introducción}

\subsection{Antecedentes}

Con la incorporación de tecnología inalámbrica en casi todos los ámbitos de la vida cotidiana, cada individuo requiere de una demanda de conectividad. Sin embargo, hay zonas alejadas de los logares donde se centraliza el servicio, y en algunos casos no es posible brindar el servicio donde la topografía del terreno lo impide. Debido al incremento en el tráfico de los canales de comunicación por el uso de internet en las redes móviles, y a que el número de consumidores ha aumentado drásticamente en los últimos años; el servicio de los proveedores tiende por obligación a modernizar sus tecnologías para brindar un mejor producto al que presenta la competencia, esto es un incremento en la tasa de usuario, se traduce a mayor velocidad, menor costo e incremento en el alcance y la cobertura. Este es el motivo de utilizar la tecnología que está en el mercado brindando un menor índice de error en la caída de sus señales; disminuir el costo de recursos tecnológicos para competir y otorgar una garantía en los enlaces punto a punto. Este esquema crea mayores ingresos en los prestadores de servicio de interconectividad del servicio y cambia el costo al uso del mismo. Por ello el avance tecnológico requiere de una infraestructura que conecte los sistemas de la ciudad en una misma red para sistemas inalámbricos [1-5].

Este costo radica en todos los insumos que forman parte de la infraestructura que se debe instalar para prestar el servicio a tasas semejantes a las que puede brindar la fibra óptica. El costo se puede estimar en ciudades donde la topografía de ciudades es más o menos plana y se instalan los postes y el tendido de cables. Sin embargo, en ciudades donde la topografía es rugosa, montañosa donde físicamente no es posible el tendido de la fibra, la conectividad inalámbrica a tasas altas es viable, por ello el presente estudio así lo muestra [6-8].

Lo anterior requiere estudios de factibilidad para obtener resultados científicos. Las oportunidades y el potencial en la construcción de infraestructuras de Internet de banda ancha en las zonas. Utilizando tecnología alternativa como Broadband Wireless que utilizan la tecnología de radiofrecuencia. Haciendo observación en las topologías de los diferentes diseños para los enlaces punto a punto, el fabricante hace hincapié que la tecnología airFiber fue diseñada para crear enlaces de alto rendimiento. Este tipo de enlaces debe ser por medio de transporte aéreo por el medio de la fibra y es soportado por diferentes técnicas de duplexado como lo son FDD (Duplexado por división de Frecuencia), TDD (Duplexado por división de tiempo) y HDD (Duplexado por División Hibrido). El fabricante muestra cómo alcanzar la mejor velocidad y la menor latencia dependiendo de las condiciones climáticas, topográficas y rango de alcance del radioenlace [9-16]. La topología que sugiere Ubiquiti Network es la de anillo, por su sencillez para un enlace punto a punto y la redundancia entre enlaces, ya que, si uno de los enlaces se cae, el sistema debe tener la capacidad de encontrar la ruta necesaria para otorgar el servicio.

Esta topología configura un punto como maestro y el otro como esclavo, así genera series de maestros y esclavos para cerrar el círculo del anillo. La Figura 1 muestra la topología de una red anillo. 


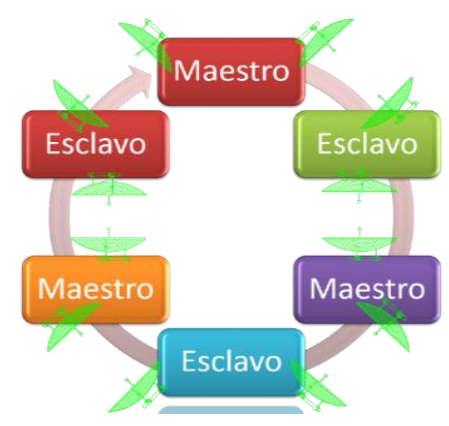

Fig. 1. Topología de anillo redundante.

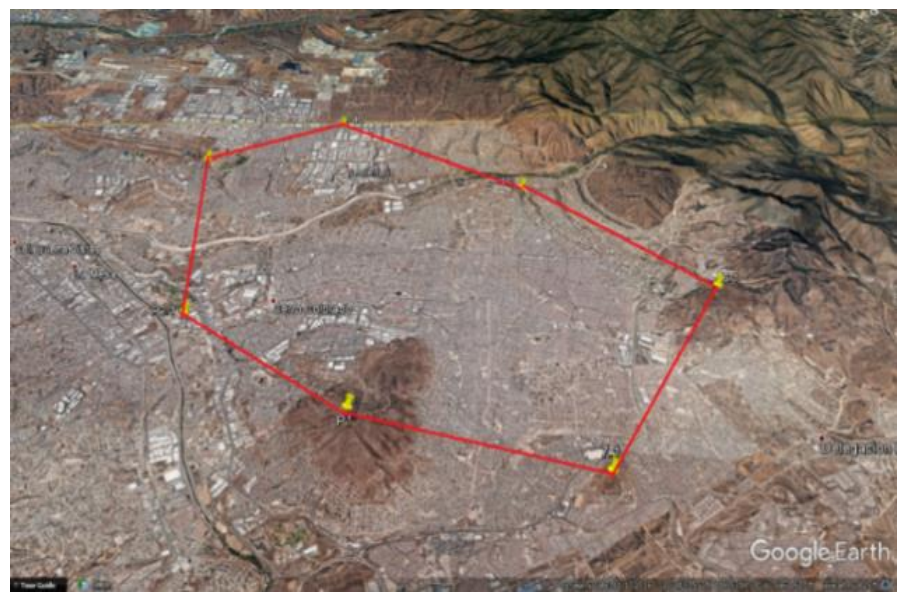

Fig. 2. Escenario anillo de los enlaces conectados.

Tabla 1. Locación de los enlaces en latitud y longitud.

\begin{tabular}{lll}
\hline Headin & Latitudes & Longitudes \\
\hline Punto 1 & $32^{\circ} 28^{\prime} 29.85^{\prime \prime}$ & $116^{\circ} 53^{\prime} 57.23^{\prime \prime}$ \\
\hline Punto 2 & $32^{\circ} 29^{\prime} 40.11^{\prime \prime}$ & $116^{\circ} 56^{\prime} 11^{\prime \prime}$ \\
\hline Punto 3 & $32^{\circ} 32^{\prime} 4.4^{\prime \prime}$ & $116^{\circ} 56^{\prime} 50.71^{\prime \prime}$ \\
\hline Punto 4 & $32^{\circ} 32^{\prime} 56.19^{\prime \prime}$ & $116^{\circ} 55^{\prime} 4.39^{\prime \prime}$ \\
\hline Punto 5 & $32^{\circ} 31^{\prime} 52.73^{\prime \prime}$ & $116^{\circ} 52^{\prime} 19.72^{\prime \prime}$ \\
\hline Punto 6 & $32^{\circ} 30^{\prime} 16.32^{\prime \prime}$ & $116^{\circ} 50^{\prime} 1.68^{\prime \prime}$ \\
\hline Punto 7 & $32^{\circ} 28^{\prime} 11.06^{\prime \prime}$ & $116^{\circ} 51^{\prime} 21.44^{\prime \prime}$ \\
\hline
\end{tabular}

Este artículo está organizado de la siguiente manera, en la Sección 2 se presenta el desarrollo de cada uno de los siete enlaces del anillo, en la Sección 3 se presenta el perfil de la trayectoria con libramiento y el nivel del margen de desvanecimiento de los radioenlaces. 
Tabla 2. Especificaciones de la Antena airFiber.

\begin{tabular}{ll}
\hline Parámetros & Valores \\
\hline Fuente de Alimentación & $24.05-24.25 \mathrm{GHz}$ \\
\hline Frecuencia de Operación & $50 \mathrm{~V}-1.2^{\mathrm{a}}$ \\
\hline Dimensiones Radio & $593 \times 768 \times 370 \mathrm{~mm}(23.35 \times 30.24 \times 14.57 ") 796 \mathrm{x}$ \\
Box & $696 \times 49.5 \mathrm{~mm}(31.34 \times 27.40 \times 1.95 ")$ \\
\hline Temperatura de Operación & $-40 \mathrm{a} 55^{\circ} \mathrm{C}$ \\
\hline Rango & $20+\mathrm{Km}$ \\
\hline Banda ancha del Canal & $100 \mathrm{Mhz}$ \\
\hline Ganancia de Tx & $33 \mathrm{dBi}$ \\
\hline Ganancia de Rx & $38 \mathrm{dBi}$ \\
\hline Polaridad & Polarización Dual \\
\hline
\end{tabular}

Tabla 3. Parámetros de los enlaces.

\begin{tabular}{llllllll}
\hline Enlace & $1-2$ & $2-3$ & $3-4$ & $4-5$ & $5-6$ & $6-7$ & $7-1$ \\
\hline $\mathrm{A}$ & 4.14 & 4.57 & 3.19 & 3.19 & 11.19 & 4.39 & 4.11 \\
\hline $\mathrm{B}$ & -63.89 & -64.75 & -61.64 & -61.64 & -72.53 & -64.40 & -63.83 \\
\hline $\mathrm{D}$ & 24 & 24 & 24 & 24 & 24 & 24 & 24 \\
\hline $\mathrm{E}$ & 38 & 38 & 38 & 38 & 38 & 38 & 38 \\
\hline $\mathrm{F}$ & 100 & 100 & 100 & 100 & 100 & 100 & 100 \\
\hline $\mathrm{G}$ & 154.03 & 153.84 & 154.49 & 153.79 & 153.81 & 153.91 & 154.03 \\
\hline $\mathrm{H}$ & -58.09 & -13.06 & 59.97 & 65.43 & 50.36 & -28.24 & 81.86 \\
\hline $\mathrm{I}$ & 211.90 & 256.93 & 329.97 & 335.43 & 320.36 & 241.75 & 188.13 \\
\hline $\mathrm{J}$ & 132.29 & 133.23 & 130.12 & 133.49 & 133.41 & 132.88 & 132.28 \\
\hline $\mathrm{K}$ & 6.95 & 8.36 & 3.69 & 8.75 & 8.62 & 7.82 & 6.93 \\
\hline $\mathrm{L}$ & -72.29 & -73.23 & -70.12 & -73.49 & -73.41 & -72.88 & -72.28 \\
\hline $\mathrm{M}$ & -59.29 & -60.23 & -57.12 & -60.49 & -60.41 & -59.88 & -59.28 \\
\hline $\mathrm{N}$ & 1.4755 & 1.4755 & 1.4755 & 1.4755 & 1.4755 & 1.4755 & 1.4755 \\
\hline & 5.8159 & 5.5085 & 6.5894 & 5.4264 & 6.5894 & 5.6224 & 5.8183 \\
\hline
\end{tabular}

\section{Desarrollo}

En un mapa mostrado en la figura 2, se localizan las coordenadas de aquellos lugares que presenten línea de vista entre sus enlaces, y liberen al menos el $60 \%$ la primera zona de Fresnel de tal forma que se cubra la mayor área posible garantizando que se mantendrá la comunicación a tasa alta.

Una vez localizados los sitios potenciales ver tabla 1 se vaciarán los datos del equipo de comunicaciones para obtener un margen aceptable del radioenlace. 
Se alimentará al programa con los datos del equipo en especial de la antena AirFiberHD24 haciendo la comparación de ciertos parámetros en el software AirLink con los presupuestos de enlace, tomando las características que otorgan los fabricantes de la antena. Para realizar el presupuesto de enlaces para un escenario de anillo se tomaron en cuenta las latitudes y longitudes de cada una de las locaciones en la región de Tijuana, que se muestran en la Tabla 2 [17].

\section{Resultados}
A. Distancia entre puntos $(\mathrm{Km})$,
B. Potencia del enlace $(\mathrm{dBm})$,
C. Frecuencia (Ghz),
D. Ganancia de la antena, estación y acceso (dBi),
E. Banda del canal (Mhz),
F. Altura de la antena desde nivel del mar (m),
G. Azimuth punto $1\left(^{\circ}\right)$,
H. Azimuth punto $2\left(^{\circ}\right)$,
I. Perdidas por espacio $(\mathrm{dB})$,
J. Margen de desvanecimiento ( $\mathrm{dBm})$,
K. Nivel de señal recibida Europea $(\mathrm{dBm})$,
L. Nivel de señal recibida Americana $(\mathrm{dBm})$,
M. $\mathrm{BW}\left({ }^{\circ}\right)$,
N. Zona de Fresnel.

Cada enlace presento condiciones especiales debido a que las alturas de los lugares para transmitir superaron el libramiento de obstrucciones de la primera zona de Fresnel, así lo demuestra cada uno de los siete enlaces. Los puntos de transmisión presentaron condiciones de rugosidad en el terreno, finalmente se concretaron cada uno de los radioenlaces, manejando márgenes de desvanecimiento por encima de los $14 \mathrm{~dB}$, lo que permite manejar toda la potencia del enlace y superar las inclemencias del tiempo para sostener la tasa alta de la transmisión.

\section{Conclusiones}

La topografía de la ciudad de Tijuana es del tipo rugosa, serrana, y con cañones, valles y mesetas, que impiden brindar calidad de servicios de telecomunicaciones a altas tasas a la población que los habita, por los altos costos que representan brindar el servicio, o la imposibilidad física del tendido e instalación de infraestructura apropiada.

El software empleado, permitió seleccionar de entre varios puntos aquellos que terminaron definiendo los siete lugares marcados, para conformar el arreglo de anillo. Cada punto del anillo logro la línea de vista sin obstrucción, con la zona despejada, libre de matorrales y construcciones futuras, que impidan el enlace en un futuro. 
El margen de desvanecimiento logrado por la potencia de transmisión, las ganancias de las antenas y la sensibilidad del receptor, garantiza que la calidad de la señal no decaiga y se presenten perdidas en el enlace a tasa alta.

Este tipo de enlaces tomo poco tiempo implementarlos, con respecto a los que deben realizar tendido de cables, y la problemática que representa cuando se rompe la fibra.

\section{Referencias}

1. Freeman, R. L.: Telecommunication system engineering. John Wiley \& Sons (2015)

2. Seybold, J.: Introduction to RF Propagation. John Wiley \& Sons (2005)

3. Longley, A. G., Rice, P. L.: Prediction of Tropospheric Radio Transmission Loss Over Irregular Terrain. ESSA Technical Report ERL 79-ITS 67 (1968)

4. Nautel: RF Toolkit Technical Resources (2012)

5. Hata, M.: Empirical Formula for Propagation Loss in Land Mobile Radio Services. IEEE Transactions on Vehicular Technolgy, 29(3), pp. 317-325 (1980)

6. UIT-R: P1238 Recomendación Datos de propagación y métodos de predicción para la planificación de sistemas de radiocomunicaciones en interiores y redes de radiocomunicaciones de área local en la gama de frecuencias de $900 \mathrm{MHz}$ a $100 \mathrm{GHz}$ (2017)

7. COST Telecommunications: Digital Mobile Radio Towards Future Generation SystemsCOST 231 Final Report (1999)

8. Walfisch, J., Bertoni, H. L.: A Theoretical Model of UHF Propagation in Urban Environments. IEEE Transactions on Antennas and Propagation, 36(12), pp. 1788-1796 (1988)

9. Recommendation ITU-R P.1406-1: Propagation Effects Relating to Terrestrial Land Mobile and Broadcasting services in the VHF and UHF Bands (2007)

10. Ikegami, F., Yoshida, S., Takeuchi, T., Umehira, M.: Propagation factors controlling mean field strength on urban streets. Antennas and Propagation, IEEE Transactions, 32(8), pp. 822-829 (1984)

11. Sarkar, T. K., Zhong, J., Kyungjung, K., Medouri, A., Salazar-Palma, M.: A survey of various propagation models for mobile communication. Antennas and Propagation Magazine, IEEE, 45(3), pp. 51-82 (2003)

12. Walfisch, J., Bertoni, H. L.: A theoretical model of UHF propagation in urban environments. Antennas and Propagation, IEEE Transactions, 36(12), pp. 1788-1796 (1988)

13. Kozono, S., Watanabe, K.: Influence of Environmental Buildings on UHF Land Mobile Radio Propagation Communications. IEEE Transactions, 25(10), pp. 1133-1143 (1977)

14. Seidel, S.Y., Rappaport, T.S.: $914 \mathrm{MHz}$ path loss prediction models for indoor wireless communications in multifloored buildings. Antennas and Propagation, IEEE Transactions, 40(2), pp. 207-217 (1992)

15. Schantz, H. G., Siwiak, K., Win, M. Z.: A Comprehensive Standardized Model for Ultrawideband Propagation Channels. Antennas and Propagation, IEEE Transactions, 54(11), pp. 3151-3166 (2006)

16. Cichon, D. J., Kürner, T.: COST 231 group final repport: Capítulo 4 Propagation Prediction Models.

17. Data sheets: The airFiber ${ }^{\circledR} 24$ is ideal for outdoor, PtP bridging and carrier-class network backhauls. https://dl.ubnt.com/datasheets/airfiber/airFiber_DS.pdf (2012) 